\title{
Clinical determinants of psychopathological outcomes after epilepsy surgery
}

$19^{\text {TH }}$ WPA WORLD CONGRESS OF PSYCHIATRY LISBON, 21-24 AUGUST, 2019

\section{Filipa Novais ${ }^{1}$, Joana Crawford ${ }^{1}$, Mafalda Andrea1, Susana Loureiro1, Luís Câmara Pestana1, Centro de Referência de Epilepsia Refractária ${ }^{1}$ \\ 1: Hospital de Santa Maria - Centro Hospitalar Lisboa Norte}

\section{Introduction}

About one-third of people with epilepsy do not respond to adequate antiepileptic drug treatment[1], therefore, they are considered to have refractory epilepsy, a chronic and debilitating condition with a great impact on patients' quality of life[2,3]. These people may be eligible for resective surgery, a procedure that is effective in the remission of seizures of about $70 \%$ of the cases[4] . After surgery, although most patients seem to achieve a general improvement, others develop de novo psychopathology or may worsen their previous psychiatric condition[5] .

The aim of this study is to determine the predictors of longitudinal changes in psychopathological symptomatology, one year after epilepsy surgery, considering clinical and demographic characteristics.

\section{Methods}

People with refractory epilepsy referred to epilepsy surgery were included in this ambispective study. Psychiatric evaluations were made before surgery and one year after the procedure. Demographic, psychiatric and neurological data were recorded. Linear regression was used to analyze longitudinal data regarding the Global Severity Index and 9 symptom dimensions of Symptom Checklist-90 (SCL-90).

\section{Results}

Seventy-six people were included. Bilateral epileptogenic zone, lack of remission of disabling seizures and Deep Brain Stimulation, targeting the anterior nucleus of the thalamus (ANT-DBS), were the most important predictors of an increase in SCL-90 scores, after surgery.

\begin{tabular}{|c|c|c|c|c|}
\hline & Engel Class II (vs Engel I) & Bilateral epileptogenesis & Multilobar epileptogenesis & ANT-DBS \\
\hline Somatization & $1.32(0.73,1.90)$ & & & \\
\hline Obsessive-Compulsive & $1.62(0.61,2.62)$ & & & \\
\hline Interpersonal sensitivity & $0.88(0.11,1.65)$ & & & \\
\hline Depression & $1.60(0.69,2.51)$ & & & \\
\hline Hostility & $0.77(0.13,1.41)$ & & $0.82(0.05,1.59)$ & $0.59(0.14,1.05)$ \\
\hline Phobic anxiety & $0.94(0.18,1.71)$ & $\begin{array}{l}\text { Left: } 0.71(0.03,1.38) \\
\text { Right: } 0.73(0.08,1.38)\end{array}$ & & $0.62(0.06,1.19)$ \\
\hline Paranoid Ideation & $1.44(0.66,2.23)$ & $\begin{array}{l}\text { Left: } 1.24(0.55,1.94) \\
\text { Right: } 1.06(0.36,1.76)\end{array}$ & & $1.17(0.62,1.73)$ \\
\hline Psychoticism & $0.97(0.22,1.73)$ & & & \\
\hline Global Severity Index & $1.19(0.54,1.85)$ & $\begin{array}{l}\text { Left: } 0.65(0.02,1.29) \\
\text { Right: } 0.65(0.02,1.28)\end{array}$ & & $0.57(0.02,1.11)$ \\
\hline
\end{tabular}

Table 1: Predictors of higher SCL-90 scores one year after surgery

\section{Conclusion}

Some individual factors may have an impact on the development or worsening of the previous psychopathology. This study identifies clinical aspects associated with greater psychological distress, after surgery. These patients may benefit from more frequent psychiatric routine assessments for early detection.

\section{Bibliography}

[1]Gooneratne IK, Green AL, Dugan P, Sen A, Franzini A, Aziz T, et al. Comparing neurostimulation technologies in refractory focal-onset epilepsy. J Neurol Neurosurg Ampamp Psychiatry 2016;87:1174 LP - 1182.

[2] Kotwas I, McGonigal A, Bastien-Toniazzo M, Bartolomei F, Micoulaud-Franchi J-A. Stress regulation in drug-resistant epilepsy. Epilepsy Behav 2017;71:39-50. doi:https://doi.org/10.1016/j.yebeh.2017.01.025.

[3]J. LM, O. EJ, Bo L, T. KE, Christine C. Serious psychological distress among persons with epilepsy based on the 2005 California Health Interview Survey. Epilepsia 2009;50:1077-84. doi:10.1111/j.1528-1167.2008.01996.x.

[4]BC J, GD C. Resective epilepsy surgery for drug-resistant focal epilepsy: A review. JAMA 2015;313:285-93.

[5]Iranzo-Tatay C, Rubio-Granero T, Gutierrez A, Garcés M, Conde R, Gómez-lbáñez A, et al. Psychiatric symptoms after temporal epilepsy surgery. A one-year follow-up study. Epilepsy Behav 2017;70:154-60. doi:10.1016/J.YEBEH.2017.02.029. 